
(I)

\title{
ECRIS : Enquête Collective Rapide d'Identification des conflits et des groupes Stratégiques...
}

Thomas Bierschenk et Jean-Pierre Olivier de Sardan

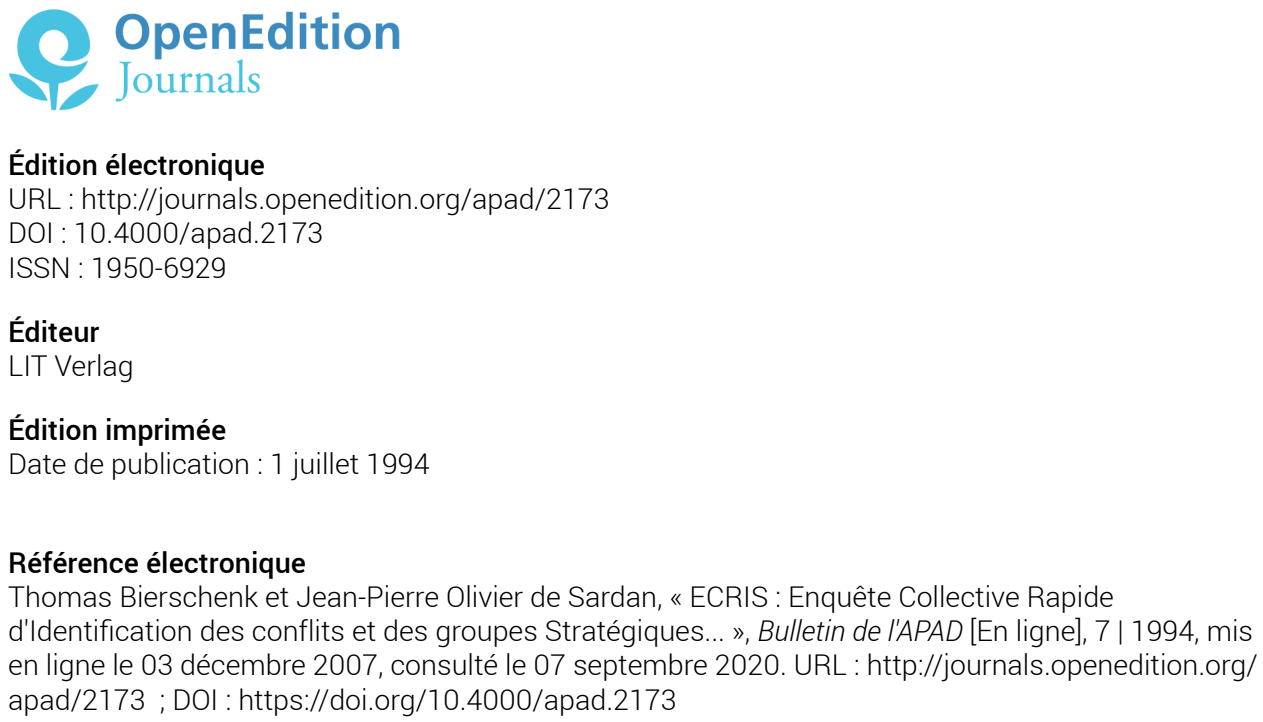

Édition électronique

URL : http://journals.openedition.org/apad/2173

DOI : $10.4000 /$ apad. 2173

ISSN : 1950-6929

Éditeur

LIT Verlag

Édition imprimée

Date de publication : 1 juillet 1994

Référence électronique

Thomas Bierschenk et Jean-Pierre Olivier de Sardan, « ECRIS : Enquête Collective Rapide d'Identification des conflits et des groupes Stratégiques... », Bulletin de l'APAD [En ligne], 7 | 1994, mis en ligne le 03 décembre 2007, consulté le 07 septembre 2020. URL : http://journals.openedition.org/ apad/2173 ; DOI : https://doi.org/10.4000/apad.2173

Ce document a été généré automatiquement le 7 septembre 2020.

Bulletin de l'APAD 


\title{
ECRIS : Enquête Collective Rapide d'Identification des conflits et des groupes Stratégiques...
}

\author{
Thomas Bierschenk et Jean-Pierre Olivier de Sardan
}

1 Ce sigle ne cache pas une technologie d'enquête "clés en main" et de courte durée qui entendrait se substituer à l'enquête socio-anthropologique de moyenne ou de longue durée. Ce n'est donc pas un nouveau "produit" à placer sur le marché aujourd'hui en expansion des études rapides, des enquêtes pressées et des évaluations au pas de course 1.

2 C'est plutôt une méthode de travail complémentaire du "terrain" classique, lequel reste indispensable et réclame une investigation individuelle relativement intensive et donc prolongée. La méthode ECRIS est simplement conçue pour précéder, impulser, et coordonner des enquêtes classiques ultérieures de chercheurs individuels dans le cadre d'une recherche comparative menée sur plusieurs sites. Mais à notre avis ECRIS permet d'améliorer considérablement le rendement et l'efficacité de ces recherches individuelles, ainsi que d'en raccourcir la durée. ECRIS permet d'autre part et peut-être surtout de fournir à ces recherches un canevas véritablement comparatif, par la mise au point collective sur le terrain d'indicateurs qualitatifs communs que chaque chercheur individuel utilisera ensuite dans son propre site de recherche.

D'autre part, et c'est dans cette perspective qu'elle a été utilisée à Dakar, c'est aussi un outil de formation, qui permet d'initier aux bases de l'enquête anthropologique sous des formes adaptées à l'évaluation de projets.

Mais contrairement à beaucoup de "méthodes" en sciences sociales, qui se veulent des "outils" indépendants des problématiques au service desquelles ils seront amenés à fonctionner (mais est-ce possible ? ${ }^{2}$ ), ECRIS est une méthode en quelque sorte à "problématique minimum incorporée". Nous nous inspirons en effet clairement d'un "point de vue" en sciences sociales que trois mots-clés résument : conflit, arène, groupe stratégique. 
5 Autrement dit, si un chercheur pense qu'un village est une communauté unie par la tradition, cimentée par le consensus, organisé par une "vision du monde" partagée, et régi par une culture commune, qu'il ne lise pas plus avant ces lignes : ECRIS ne lui sera d'aucune utilité... Nous pensons à l'inverse qu'un village est une arène, traversée de conflits, où se confrontent divers "groupes stratégiques"...

6 Conflit, arène, groupe stratégique : il convient d'expliquer ces trois concepts, où nous les avons pris, et dans quel sens nous les utilisons.

Conflit

7 Les premiers travaux en anthropologie africaniste qui aient systématiquement abordé la réalité sociale par le biais des conflits sont ceux de l'Ecole de Manchester, dont l'emblème est sans doute Gluckman, avec un titre d'ouvrage significatif : "Custom and conflict in Africa" (Gluckman, 1956). Mais l'usage par Gluckman de la notion de conflit renvoie à au moins trois niveaux différents d'analyse qu'il est utile de désagréger.

D'une part, un constat empirique : les sociétés, toutes les sociétés, sont traversées de conflits. Le conflit est donc un élément inhérent à toute vie sociale.

D'autre part, une analyse structurelle: les conflits renvoient à des différences de positions. Autrement dit les sociétés, aussi petites soient-elles, et aussi dépourvues soient-elles de formes institutionnalisées de "gouvernement", sont divisées et clivées. Ces divisions et ces clivages sont entretenus par des "coutumes", c'est-à-dire des normes, des règles morales, des conventions (on pourrait aussi dire des codes culturels). Les conflits expriment donc des intérêts différents liés à des positions sociales différentes et sont culturellement structurés.

Enfin, un postulat fonctionnaliste: les conflits, qui semblent vouer les sociétés à l'émiettement ou à l'anarchie, concourent au contraire à la reproduction sociale et au renforcement en dernière analyse de la cohésion sociale : ils permettent de maintenir le lien social.

11 Nous récusons le postulat fonctionnaliste, nous amendons l'analyse structurelle, et nous reprenons le constat empirique.

On comprendra facilement pourquoi le postulat fonctionnaliste est aujourd'hui dépassé, et pourquoi le constat empirique est au contraire toujours valable, sans qu'il soit besoin de développer ici ces deux argumentations. Par contre on peut expliquer brièvement en quoi l'analyse structurelle doit être amendée (en suivant d'ailleurs les traces d'élèves de Gluckman comme Turner : Turner, 1957) : il est vrai que bien souvent les conflits renvoient à des positions différentes dans la structure sociale. Mais il convient de souligner l'existence d'une "marge de manœuvre" pour les individus (cf. Long, 1989. qui a introduit dans la sociologie et l'anthropologie du développement la problématique de l'Ecole de Manchester). Un conflit entre personnes ou entre groupes n'est pas que l'expression d'intérêts "objectifs" opposés, mais aussi l'effet de stratégies personnelles, plus ou moins liées à des réseaux et organisées en alliances. L'analyse structurelle doit être complétée par une analyse stratégique.

Mais nous privilégions surtout la dimension heuristique du repérage et de l'étude des conflits, et c'est celle-ci qui est pour ECRIS fondamentale: les conflits sont un des meilleurs "fils directeurs" qui soient pour "pénétrer" une société et en révéler tant la structure que les normes ou les codes. Postuler l'existence d'un consensus est une hypothèse de recherche beaucoup moins puissante et productive que de postuler l'existence de conflits. Les conflits sont des indicateurs privilégiés du fonctionnement 
d'une société locale. Ce sont aussi des indicateurs du changement social, particulièrement pertinents pour une anthropologie du développement.

Identifier les conflits, c'est aussi un moyen d'aller au-delà de la façade consensuelle et de la mise en scène en direction de l'extérieur que les acteurs d'une société locale proposent souvent à l'intervenant ou au chercheur extérieur.

Il ne faut donc voir, dans notre approche d'une société par ses conflits, ni la recherche du conflit pour le conflit, ni la volonté de privilégier les conflits sur toute autre forme de sociabilité, ni le refus de prendre en compte les consensus ou les codes communs. Notre hypothèse n'est qu'une hypothèse méthodologique, bien souvent vérifiée, selon laquelle le repérage et l'analyse des conflits sont des pistes de recherche fructueuses, qui font gagner du temps, et qui évitent certains des pièges que les sociétés ou les idéologies tendent aux chercheurs.

Il restera bien sûr à hiérarchiser les conflits, à comprendre leurs liens mutuels éventuels. Autrement dit il restera à les analyser. Ceci est une autre affaire, qui sort en quelque sorte du cadre d'ECRIS proprement dit. ECRIS n'entend pas se substituer à la nécessaire théorisation de tout travail empirique.

Arène

17 C'est peut-être dans le contexte des analyses de Bailey que le terme, fréquemment utilisé dans la littérature anglo-saxonne, est le plus significatif, bien qu'il ne soit jamais explicitement défini (Bailey, 1969). Bailey voit la vie politique, nationale comme locale, en termes de "jeu", où se confrontent et s'affrontent les acteurs sociaux, autour de leaders et de factions. L'arène est au fond l'espace social où prennent place ces confrontations et affrontements.

La notion d'arène peut utilement être rapprochée de notions voisines, celle anglo-saxonne de social field (espace transversal où coexistent des institutions et des rôles multiples), ou celle de "champ", largement utilisée par Bourdieu (cf. par exemple Bourdieu 1966, 1971). Chez Bourdieu, "champ" est une notion polysémique, jamais clairement définie, qui oscille entre plusieurs acceptions. C'est à la fois un "marché" (au sens métaphorique) où les acteurs dotés de "capitaux" divers (capital économique, symbolique, social...) sont en concurrence, à la fois un certain type de structure sociale autonomisée (des institutions, des agents spécialisés, un langage) et à la fois un espace de jeu et d'enjeux relevant d'un rapport de forces entre groupes sociaux. Mais dans tous les cas, "champ" reste une notion à la fois très "macro" et abstraite. Nous pourrions dans cette perspective parler du "champ du développement", afin de décrire ces institutions spécifiques, ce langage particulier, ce marché inégalement structuré et inégalement concurrentiel où se confrontent des idéologies, des salaires, des compétences, des institutions, des symboles, etc...

19 Arène évoque à la fois une échelle plus restreinte et une plus claire conscience des affrontements chez les acteurs eux-mêmes. Une arène, au sens où nous l'entendons, est un lieu de confrontations concrètes d'acteurs sociaux en interaction autours d'enjeux communs. Un projet de développement est une arène. Le pouvoir villageois est une arène. Une coopérative est une arène. Arène a un contenu empirique plus fort que champ. C'est bien sûr une notion à géométrie variable, dont l'extension et la forme varient selon les enjeux sociaux qui permettent de la définir. C'est surtout une notion à valeur exploratoire.

Groupe stratégique 
20 C'est vers Evers (Evers \& Schiel, 1988) que nous nous sommes cette fois tournés pour emprunter le concept de groupe stratégique. Chez ce sociologue allemand, il s'agit de proposer une alternative à la catégorie de "classe sociale", trop figée, trop mécanique, trop économique, trop dépendante d'une analyse marxiste en termes de "rapports de production". Les groupes stratégiques apparaissent ainsi comme des agrégats sociaux plus empiriques, à géométrie variable, qui défendent des intérêts communs, en particulier par le biais de l'action sociale et politique.

21 Cette perspective plus pragmatique qui, au lieu de définir a priori les critères de constitution de groupes sociaux les déduit de l'analyse des formes d'action nous semble intéressante. Mais pour Evers, les groupes stratégiques restent au fond au même niveau "macro" que les classes sociales auxquelles ils se substituent. Ils n'interviennent qu'à l'échelle de l'arène nationale, ou de la société globale. Nous estimons pour notre part que c'est surtout au niveau de la société locale que le concept est opératoire, lorsqu'il est lié à l'observation des formes d'interaction entre acteurs.

Il reste le problème de savoir si les groupes stratégiques sont des groupes "réels", plus ou moins "en corps", dotés de normes communes, de formes d'action collective ou de procédures de concertation, ou si ce sont plutôt des agrégats artificiels construits par l'analyste. Notre position est sur ce point pragmatique : nous considérons au départ le groupe stratégique comme une hypothèse de travail du chercheur, comme une sorte de "groupe virtuel" qui nous aide à penser la convergence des stratégies entre certains individus dont on peut supposer qu'ils partagent une même position face à un même "problème". Autrement dit, face à un "problème" donné dans un contexte social donné, il n'y a pas une infinité d'attitudes et de comportements : on constate un nombre fini d'attitudes et comportements, qui semblent liés aux relations respectives que les acteurs entretiennent avec ce "problème", autrement dit à leurs positions sociales en rapport à ce problème. C'est un des objets mêmes de la recherche que de déterminer in fine si les groupes stratégiques qui ont été posés comme hypothèse de départ ont ou non une existence "réelle", autrement dit si les acteurs ayant une position commune partagent ou non des formes particulières d'interaction ou de concertation (de façon informelle - réseau, affiliation, allégeance - ou formelle - institution, appartenance, organisation). Contrairement aux définitions sociologiques classiques des groupes sociaux, les "groupes stratégiques" (virtuels ou réels) ne sont pas pour nous constitués une fois pour toutes et pertinents quels que soient les problèmes : ils varient selon les problèmes considérés, c'est-à-dire selon les enjeux locaux. Parfois ils renverront à des caractéristiques statutaires ou socio-professionnelles (sexe, caste, métier, etc...), parfois à des affiliations lignagères ou à des réseaux de solidarité ou de clientèle, parfois à des parcours biographiques et des stratégies individuelles.

Chaque acteur social peut évidemment, selon les contextes ou les circonstances, appartenir potentiellement à des groupes stratégiques différents, en fonction du répertoire de rôles qui est le sien. Il n'y a donc pas de frontières rigides entre les groupes stratégiques. C'est le processus de l'enquête elle-même qui doit permettre de complexifier le schéma de départ. La notion de groupe stratégique reste essentiellement d'ordre empirique et méthodologique. Elle suppose simplement que dans une collectivité donnée tous les acteurs n'ont ni les mêmes intérêts, ni les mêmes représentations, et que, selon les "problèmes", leurs intérêts et leurs représentations s'agrègent différemment, mais pas n'importe comment. On peut donc faire des hypothèses sur ce que sont les groupes stratégiques face à un "problème" donné : 
l'enquête montrera évidemment si ces hypothèses sont justes ou non, s'il faut recomposer autrement les groupes stratégiques, quelle est leur existence sociale, et s'ils peuvent ou non se transformer en stratégies collectives et en alliances...

La procédure de ECRIS

ECRIS se déroule en six phases. On notera que la démarche est un continuel va-et-vient entre phases individuelles et phases collectives, à la différence de l'enquête ethnographique classique qui privilégie la recherche individuelle de longue durée, et à la différence aussi des méthodes d'enquêtes accélérées de type RRA qui privilégient l'enquête collective de courte durée. On notera également que la méthode propose avant tout des cadres d'investigation et des repères collectifs, à la différence encore de l'enquête ethnographique classique où le chercheur organise comme bon lui semble son travail et son recueil de données, et à la différence aussi des méthodes d'enquêtes accélérées de type RRA, qui fournissent des boîtes à outils standardisées.

Une enquête individuelle de repérage

Il s'agit là de préparer rapidement (un à deux jours sur chaque site de recherche) le travail d'équipe à venir en identifiant sommairement les principaux enjeux locaux (en fonction du thème de la recherche bien sûr), afin de pouvoir prédéterminer des groupes stratégiques (c'est-à-dire proposer des groupes stratégiques provisoires pour l'enquête collective à venir), regroupant des catégories d'acteurs dont on peut présumer qu'ils partagent un même rapport global à ces enjeux.

Si le thème de la recherche est, mettons, l'évaluation d'un projet de développement local, l'enquête préliminaire relèvera par exemple l'existence d'enjeux fonciers liés au projet, de conflits agriculteurs/éleveurs, de rivalités entre deux grandes familles aristocratiques, ainsi que l'exclusion des femmes des bénéfices du projet. On pourra alors proposer comme groupes stratégiques provisoires : (1) les simples agriculteurs, (2) les simples éleveurs, (3) les deux familles aristocratiques, (4) les intervenants extérieurs (ONG, services techniques), (5) les femmes.

Un séminaire de préparation

27 Ce séminaire (deux jours) doit familiariser les participants avec la problématique et la méthode, faire le point de la documentation sur les sites de recherche, et proposer une série d'indicateurs qualitatifs provisoires susceptibles de guider les recherches individuelles ultérieures (on ne peut évidemment proposer des indicateurs standards, chaque thème d'enquête nécessitant le "bricolage" d'indicateurs spécifiques).

On pourrait proposer, pour poursuivre avec l'exemple précédent, comme indicateurs provisoires: l'histoire et la typologie des projets s'étant succédé dans le village, l'analyse d'un processus de décision local lié au projet actuel, la biographie de quelques acteurs centraux du projet, la description d'une assemblée générale, l'inventaire des lieux de débats et de discussion dans le village...

L'enquête collective

Le principe de base de ECRIS est le suivant : l'ensemble de l'équipe d'enquêteurs tourne successivement sur chaque site et reste deux jours sur chaque site. Sur un site donné, les enquêteurs se divisent en plusieurs groupes d'enquêteurs (2 à 3 personnes maximum par groupe). Chaque groupe d'enquêteurs se focalise pendant les deux jours sur un groupe stratégique local et un seul. Il n'enquête que sur des personnes relevant du groupe stratégique qui lui a été affecté. La composition des groupes d'enquêteurs change d'un site à l'autre... 
confronter à l'approche d'un problème via la notion de groupe stratégique, ànsi que de se confronter à la variété et à la relativité des groupes stratégiques. On ne considère pas le groupe stratégique comme un "vrai" groupe, un "collectif' ou un groupe "en corps" (corporate group). On ne suppose pas que le groupe stratégique ait une position commune établie. Il n'est pas question de "focus group" : si certains entretiens peuvent être collectifs (en général parce que les circonstances l'imposent, et qu'un entretien individuel se transforme vite en entretien collectif informel dès lors qu'il n'est pas secret...), on privilégie plutôt les entretiens individuels, avec des personnes aussi variées que possible à l'intérieur du groupe stratégique affecté à un groupe d'enquêteurs.

31 Si sur le site retenu il y a 10 enquêteurs, on fera donc 5 groupes d'enquêteurs de chacun 2 personnes. Un de ces groupes enquêtera par exemple uniquement auprès des femmes. Mais il ne réunira pas les femmes du village ou ne convoquera pas leurs responsables. Il ira voir successivement femmes de chef et simples paysannes, vieilles femmes et jeunes femmes, responsables associatives et femmes marginalisées, etc. La consigne principale est simple :
1. Il s'agit d'identifier au fil de l'enquête le maximum possible de conflits et de contradictions, y compris ceux où les interlocuteurs ne sont pas impliqués directement.

Par exemple les entretiens avec des femmes permettront de préciser non seulement les conflits entre femmes et hommes à propos de la commercialisation, mais aussi d'évoquer leurs points de vue sur les conflits entre éleveurs et agriculteurs, ou entre les deux lignages aristocratiques, ainsi que de repérer de nouveaux conflits (autour des appartenances religieuses ou politiques, ou à propos du renouvellement du bureau de la coopérative, ou en raison de soupçons de détournement..).

On peut y ajouter deux consignes complémentaires :

2. Tenter de comprendre le plus possible "de l'intérieur" la relation que les membres de ce groupe stratégique entretiennent avec ce qui constitue le thème de la recherche ainsi que leurs perceptions des autres groupes, et essayer de décomposer le groupe stratégique en diverses composantes ayant des comportements ou des discours communs, et se différenciant des autres composantes.

Quelles visions et quels usages les femmes d'agriculteurs ont-elles du projet de développement? Que pensent-elles des intervenants extérieurs et du rôle du sous-préfet?

Les discours tenus parmi les femmes d'éleveurs sont-ils différents? Jeunes femmes et vieilles femmes semblent-elles avoir les mêmes positions, les mêmes appréciations?

3. Approfondir les indicateurs qualitatifs provisoires (mis au point lors du séminaire de préparation) et chercher des domaines où ils pourraient être mis en œuvre.

La "décision" locale dont il serait intéressant de faire l'histoire pourrait être le renouvellement $\mathrm{du}$ bureau...; tels et tels acteurs pourraient faire l'objet d'une biographie...; le baobab au centre du village et le domicile du chef le samedi matin lorsqu'il rend justice sont les principaux lieux de débats à observer, etc.

Mais il serait aussi intéressant de rajouter parmi les indicateurs un recensement des diverses associations, des membres de leurs bureaux et des liens de parenté entre ceux-ci... 

celui-ci, puisque la confrontation avec les résultats obtenus auprès des autres groupes, comme le fait de changer de groupe sur le site suivant, permettent de relativiser les points de vue. L'entrée par les groupes stratégiques a l'avantage de fournir une saisie du social dans sa diversité, tout en s'intéressant le plus possible "de l'intérieur" à chacun des éléments de cette diversité. L'enquêteur est confronté à une pluralité de logiques sociales, et doit prendre au sérieux chacune d'entre elles. Ceci est beaucoup plus difficile à mettre en œuvre dans une enquête uniquement individuelle, où le chercheur est sans cesse menacé de s'identifier à un sous-groupe ( $d$ 'être "encliqué") et a des difficultés à passer d'un point de vue local à un autre : de ce fait il risque soit de rester extérieur aux divers points de vue locaux, soit de s'enfermer dans un seul d'entre eux.

Un séminaire de bilan d'enquête collective

Celui-ci (deux jours) a trois objectifs :

- d'une part l'élaboration finale des indicateurs qualitatifs communs, en quelque sorte testés au cours de l'enquête collective, qui serviront à chaque chercheur de points d'appuis pour son enquête personnelle

- d'autre part la détermination des pistes de travail propres à chaque site

- enfin, un premier essai comparatif, tentant de dégager à partir des différents sites les points communs comme les spécificités de chacun, les lignes de force, les principales hypothèses.

Bulletin de l'APAD, 7 | 1994 
Les recherches individuelles sur chaque site

Désormais la phase de travail de terrain individuel complémentaire est considérablement déblayée et sérieusement mise sur les rails. Il n'y a plus de procédure unique qui puisse être proposée: ECRIS lègue à chacun une série d'indicateurs communs, et une série de pistes particulières. Ce travail individuel ne peut avoir de durée standard. Tout dépend en effet des sujets explorés. Certains peuvent demander des enquêtes complémentaires individuelles fort courtes de l'ordre d'une semaine (l'analyse d'une coopérative villageoise ou l'évaluation d'un petit projet local), d'autres des enquêtes complémentaires individuelles nettement plus longues de l'ordre de plusieurs mois (l'évaluation de projets intégrés ou l'étude des formes de pouvoir local).

Le séminaire final

Préparé par des rapports rédigés à propos de chaque site, il est entièrement consacré (sur deux jours) à l'analyse comparative, à travers l'interprétation des données locales, les résultats obtenus à travers les indicateurs qualitatifs, et le débat autour des hypothèses proposées.

Conclusion

4 ECRIS a sans doute une pertinence particulière en termes de socio-anthropologie du développement, et peut avoir également une fonction d'aide à l'évaluation, pour deux raisons fondamentales :

- En tant que méthode d'analyse comparative sur plusieurs sites, ECRIS correspond bien aux besoins d'analyses liées à la préparation, au suivi ou au bilan d'opérations de développement. En particulier l'élaboration au coup par coup d'indicateurs qualitatifs non standardisés, qui font souvent défaut dans un monde du développement dominé par des indicateurs chiffrés et standards le plus souvent non fiables, est un atout important.

- Les concepts de conflit, d'arène et de groupe stratégique sont particulièrement adaptés à l'insertion d'un projet de développement dans des sociétés locales.

Cette méthode est une méthode de recherche à part entière et non un sous-produit simplifié destiné à des enquêtes sommaires. Mais elle peut contribuer à mettre les compétences de la recherche socio-anthropologique au service d'opérateurs du développement soucieux d'une meilleure compréhension des processus sociaux qui sont à l'œuvre lorsque les actions de développement se confrontent aux populations destinataires: en effet ECRIS introduit quelques outils conceptuels, quelques contraintes méthodologiques et quelques gains d'efficacité et de temps qui peuvent aider à réduire sérieusement l'écart entre les habitudes des chercheurs et les demandes des institutions de développement.

C'est aussi une méthode de formation, que ce soit formation à la recherche pour de jeunes chercheurs, ou initiation à la recherche socio-anthropologique pour des opérateurs de développement. En ce dernier cas seules les 4 premières phases sont utilisées.

Cette méthode est en pleine évolution, et se veut d'ailleurs fondamentalement évolutive. Il serait contraire à son esprit même qu'elle débouche sur un mode d'emploi standard incitant à une reproduction à l'identique. Nous avons proposé ici pour la première fois une tentative de formalisation de ce qui est largement un savoir-faire issu de pratiques bricolées et expérimentales. Nous espérons que ECRIS continuera à s'adapter ainsi d'un objet de recherche à un autre, d'un terrain à un autre, d'une équipe à une autre. Cette souplesse est une des conditions de sa réussite. 


\section{BIBLIOGRAPHIE}

Bailey F., 1969, Stratagems and spoil. A social anthropology of politics, London : Basil Blackwell (trad. fr. 1971 : Les règles du jeu politique).

Bierschenk Th., 1988, Development projects as arenas of negociation for strategie groups. A case study from Benin, Sociologica Ruralis, 28\{2-3) : 146-160.

Bierschenk Th., Sauer P. \& Schafft H., 1989, Planspiel zur entwicklungspolitischen Projektpraxis. Ein Beitrag zur Entdamonosierung der Entwicklungshilfe, Berlin : Das Arabische Buch.

Boiral P., Lantéri J.-F., Olivier de Sardan J.-P. (ed), 1985, Paysans, experts et chercheurs en Afrique Noire. Sciences sociales et développement rural, Paris : Karthala.

Bourdieu P., 1966, Champ intellectuel et projet créateur, Les Temps Modernes, 246.

Bourdieu P., 1971, Genèse et structure du champ religieux, Revue Française de Sociologie, 12 : 295-334.

Evers H. \& Schiel T., 1988, Strategische Gruppen, Berlin : Reimer.

Glaser \& Strauss A., 1973, The discovery of grounded theory. Strategies for qualitative research, Chicago : Eldin.

Gluckman M., 1956, Custom and conflict in Africa, London : Blackwell.

Long N. (ed), 1989, Encounters at the interface. A perspective on social discontinuities in rural development, Wageningen : Agricultural University.

Olivier de Sardan J-P., 1984, Les sociétés songhay-zarma. Chefs, esclaves, guerriers, paysans..., Paris : Karthala.

Olivier de Sardan, J.-P., 1993, Le développement comme champ politique local, Bulletin de l'APAD, $6: 11-18$. .

Turner V., 1957, Schism and continuity in an African society, Manchester : Manchester University Press.

\section{NOTES}

1.Cette méthode a une préhistoire et une histoire. Dans la préhistoire, il faut ranger les travaux antérieurs de recherche des auteurs (cf. Boiral, Lantéri \& Olivier de Sardan, 1986 ; Olivier de Sardan, 1984, 1993 ; Bierschenk, 1988, 1989), qui ont développé de façon indépendante et sur des terrains différents des problématiques proches, dont on trouve ici l'écho direct. On peut aussi mentionner les expériences pédagogiques menées par chacun. Quant à l'histoire, elle est encore fraîche. Nous avons en effet testé cette méthode en deux occasions. Une première expérience a été menée à Dakar, en décembre 1991, au cours d'un stage de formation du Ministère français de la Coopération à destination de coopérants français et de cadres africains (en collaboration avec Elisabeth Paquot du GRET et avec l'aide de chercheurs de l'ENEA et de ENDA-GRAF). Un ouvrage à caractère méthodologique doit paraittre à la Documentation Française pour rendre compte des acquis de ce stage. Une seconde 
expérience a été menée au Bénin, en mars 1993, dans le cadre d'un programme de recherche sur le pouvoir local (financé par le Ministère français de la Recherche, la DFG, le Ministère allemand de la Coopération, et la Mission française de Coopération et d'Action Culturelle au Bénin, en collaboration avec Marc Lévy du GREf, et avec l'aide de chercheurs de l'Université Nationale du Bénin). Un ouvrage collectif portant sur les résultats de cette recherche est en préparation.

2.On peut en effet se demander si toute méthode ne suppose pas un certain type d'affinité sélective avec une problématique ou un "point de vue" sur le social. Mieux vaut selon nous expliciter cette parenté que de l'ignorer ou de la cacher. 